\title{
Optimal Placement and Sizing of Distributed Generation in Distribution Power System using Dragonfly Algorithm
}

\author{
Alpa A. Amin ${ }^{1}$, Rajan K. Patel ${ }^{2}$, Mihir R. Vasavada ${ }^{3}$ \\ Student, Electrical Department, L.D. College of Engineering, Ahmedabad, India ${ }^{1,2}$ \\ Assistant Professor, Electrical Department, L.D. College of Engineering, Ahmedabad, India ${ }^{3}$
}

\begin{abstract}
In this article recently developed novel swarm intelligence optimization technique is proposed called dragonfly algorithm based on swarming behaviors of dragonflies to solve distributed generation placement and sizing problem on IEEE 33 bus test case. The location and size of DGs are the control parameters, in order to minimize the total active power losses and voltage deviation and tomaximize voltage stability margin, using multi-objective function as weighted sum of each objective in distribution network. Three cases are considered where a new objective function is introduced in each case and combined multi-objective problem is solved. The results of optimization problems are compared to that found in literature.
\end{abstract}

Keywords: Distributed Generation, Dragon fly algorithm, IEEE 33 bus test case.

\section{INTRODUCTION}

DG technology has been there in market for a while, consumer are installing small generation sets near their area for either as main or backup unit. Distributed generation can be defined as "The integrated use of small generation units directly connected to a distribution network or on the consumer side of the meter". The total cost of distributed generation is greatly dependent on the electrical power losses [1]. The better alternative is to use renewable sources of energy to improve power losses and costs, although other important issues are also enhanced such as voltage magnitudes and network congestion. However, determining the best location and size of renewable energy generators can be sometimes a challenging task due to a large number of possible combinations in the search space. DG planning techniques can be classified as: deterministic modelling and stochastic modelling. In Deterministic models load of network is assumed to be constant whereas probabilistic approach uses statistics and probability theory.It is observed that DG planning and placement problem are highly non-linear, non-convex complex problem. Several methods were recommended in the literature to solve these kinds of problems. Rau \& Wan [3] and AlHajri\& El-Hawary [4] used deterministic approach to allocate distributed resources in electrical networks to minimize power losses, line loadings, and reactive requests.Optimal DG sitting and sizing problem to minimize a weighted objective function consisting of power losses, voltage variation, active and reactive powers on the bus lines was solved byVallem\&Mitra [5] and Kamaliniaet al. [6] . In their mode they used Genetic algorithms, simulated annealing and data envelopment analysis (DEA).

WanxingSheng[7] proposed an improved non-dominated sorting genetic algorithm- II (INSGA-II) proposed for optimal planning of multiple distributed generation (DG) with multi-objective functions that take minimum voltage deviation, minimum line loss, and maximal voltage stability margin into consideration apart from this, a trade off based fuzzy set theory is used to determine the best compromise solution from the Pareto-optimal set. Yuan Liu, Yunhua Li [8] used harmony search (HS) with the fast non-dominated sorting approach, which lead to formation of multi-objective harmony search (MOHS) is used on IEEE 33 bus test case.Santanu Roy [9] used cuckoo optimization algorithm (COA) in order to find the optimal location of DG. Bat algorithm and PSO was used to solve minimization of power loss problem by John E. Candelo and Helman Hernández Riaño [10] and the method was tested on IEEE 14, 30 and 33 bus system, voltage level and power losses were improved for all cases. Khattam et al [11] used perspective of Distribution Company for optimal sizing and siting decisions for DG capacity.Cost wise benefit analysis on hourly basis was done in this method. Tabusearchmethod was used by Nara et al in [12]. In [13], the cost function is all about minimizing the money invested on DG and its operating cost using the same technique.In 2016Mirjalili, Seyedali [14] proposed a novel swarm intelligence optimization technique called dragonfly algorithm (DA), inspired by the static and dynamic swarming behaviours of dragonflies in nature. Both exploration and exploitation are designed by modelling static and dynamic behaviour of dragonflies. 


\section{IJIREEICE \\ International Journal of Innovative Research in Electrical, Electronics, Instrumentation and Control Engineering \\ ISO 3297:2007 Certified \\ Vol. 5, Issue 4, April 2017}

In this paper, Dragon fly algorithm is applied onDG planning and placement problems, three main objective functions used for the purpose are: total active power losses, voltage deviation and voltage stability margin improvement. The method is tested on IEEE 33 bus test case and results are compared with that found in literature.

Rest of the paper is organized with introduction of distributed generation planning and placement problem formulation in Section II, Section III illustrates the Dragon fly optimization technique and results of programs on IEEE 33 bus test case arepresented in Section IV, finally conclusions are drawn in section V.

\section{PROBLEM FORMULATION}

DG placement and sizing problem deals with adjusting controlling parameters such as location and size of DG, so that we obtain the best operation point, but one thing that needs to be taken care of is, while obtaining optimal point the constraints are not breached. Three objective functions considered in literatureare 1) reducing system line losses; 2) reducing voltage deviation; and 3) increasing voltage stability margin when DG units are considered in the distribution network (DN).

A. Minimization of line losses

By placing DG sets of right size at right place will lead to reduction in current injection into the network which inturn reduces the losses this objective function is as:

$$
\min \mathrm{f}_{1}(\mathrm{x})=\min \sum_{(\mathrm{i}, \mathrm{j}) \in \mathrm{B}} \mathrm{g}_{\mathrm{ij}}\left(\mathrm{V}_{\mathrm{i}}^{2}+\mathrm{V}_{\mathrm{j}}^{2}-2 \mathrm{~V}_{\mathrm{i}} \mathrm{V}_{\mathrm{j}} \cos \theta_{\mathrm{ij}}\right)
$$

Where, Bisnumber of branches of network, $(i, j)$ represents two nodes of a branch, $V_{i}$ and $V_{j}$ are voltage magnitudes of nodes $i$ and $j$, respectively. $g_{i j}$ is the conductance between nodes $i$ and $j$.

B. Minimization of voltage deviation

Voltage deviation is an indicator of the power quality of the system, the minimization of voltage deviation can help guarantee a better voltage level in distribution power systems. It also helps us to determine the health and security of the network. The function can be written as:

$$
\min f_{2}(x)=\min \sum_{i=0}^{N}\left(\frac{V_{i}-V_{i}{ }_{i}^{\text {spec }}}{V_{i}^{\text {max }}-V_{i}{ }^{\text {in }}}\right)^{2}
$$

Where, $V_{i}{ }^{\max }$ and $V_{i}{ }^{\min }$ are the upper and lower limits at the $i^{\text {th }}$ bus, respectively. $\mathrm{N}$ is the number of buses. $\mathrm{V}_{\mathrm{i}}$ is the voltage magnitude at the $i^{\text {th }}$ bus, and $V_{i}^{\text {spec }}$ is the specified voltage magnitude. The difference is squared to avoidnegative values of voltage deviation.

C. Maximization of Voltage stability margin

L-index, is chosen as the indicator for voltage stability index. Voltage stability margin is an indicator of the system security, it measures the security level of the distributed network there are several indices. The L-index of branch can be expressed as follows:

$$
\mathrm{L}_{\mathrm{ij}}=\frac{4\left[\left(\mathrm{P}_{\mathrm{i}} \mathrm{X}_{\mathrm{ij}}-\mathrm{Q}_{\mathrm{j}} \mathrm{R}_{\mathrm{ij}}\right)^{2}+\left(\mathrm{P}_{\mathrm{j}} \mathrm{R}_{\mathrm{ij}}+\mathrm{Q}_{\mathrm{j}} \mathrm{X}_{\mathrm{ij}}\right)_{\mathrm{i}}{ }^{2}\right]}{\mathrm{V}_{\mathrm{i}}{ }^{2}}
$$

Where, $L_{i j}$ indicates the extent of branch voltage stability. The branch voltage will be instable if the value of $L_{i j}$ is large. Voltage instability of the network is determined by the most instable branch, and its expression is as

$$
\mathrm{L}=\max \left(\mathrm{L}_{1}, \mathrm{~L}_{2}, \ldots, \mathrm{L}_{\mathrm{N}-1}\right)
$$

The value of L-index is in between 0 (no load of system) to 1 (voltage collapse). The bus with the highest L-index will be the most vulnerable bus hence,we can find areas where we need to provide critical support. So to maximise the voltage stability margin our function becomes:

$$
\min f_{3}(x)=\min L
$$

D. Constraints in optimization problem

As mentioned earlier the concern for adjusting parameters is such that network constraints are not violated and thus three main types of constraints are dealt with.

1. Equality constraints: These are power flow equations of the system, 


$$
\begin{gathered}
P_{D G i}-P_{d i}=V_{i} \sum_{j=1}^{N} V_{j}\left(G_{i j} \cos \theta_{i j}+B_{i j} \sin \theta_{i j}\right) \\
Q_{D G i}-Q_{d i}=V_{i} \sum_{j=1}^{N} V_{j}\left(G_{i j} \sin \theta_{i j}-B_{i j} \cos \theta_{i j}\right)
\end{gathered}
$$

$\mathrm{P}_{\mathrm{DGi}}$ and $\mathrm{Q}_{\mathrm{DGi}}$ - active and reactive generation outputs

$\mathrm{P}_{\mathrm{di}}$ and $\mathrm{Q}_{\mathrm{di}}-$ active and reactive loads at nodei

2. Inequality constraints: There is the limit on amount of power a generator can generate and that has been limited by Generator limits.

$$
\begin{aligned}
\mathrm{P}_{\mathrm{DGi}}^{\min } & \leq \mathrm{P}_{\mathrm{DGi}} \leq \mathrm{P}_{\mathrm{DGi}}^{\max } \\
\mathrm{Q}_{\mathrm{DGi}}^{\min } & \leq \mathrm{Q}_{\mathrm{DGi}} \leq \mathrm{Q}_{\mathrm{DGi}}^{\max }
\end{aligned}
$$

The terminal voltage of any bus must remain within the specified range $( \pm 5 \%)$ therefore voltage constrain given by equation below also has to be incorporated.

$$
V_{i}^{\min } \leq V_{i} \leq V_{i}^{\max }
$$

Similarly there are line flow limits also known as thermal limits of the line which has to be taken care of given by equation below:

$$
S_{\mathrm{ij}}^{\min } \leq \mathrm{S}_{\mathrm{ij}} \leq \mathrm{S}_{\mathrm{ij}}^{\max }
$$

3. Distribution generation capacity constraint: Every country has a limit on the penetration of DG for a distribution system to ensure reliability. If we assume the maximum DG penetration factor is $25 \%$, then the maximum injected DG capacity should be less than $25 \%$ of the total active power load in DN, that is:

$$
\sum_{\mathrm{i}=1}^{\mathrm{N}_{\mathrm{DG}}} \mathrm{P}_{\mathrm{DGi}} \leq 0.25 \sum_{\mathrm{i}=1}^{\mathrm{N}} \mathrm{P}_{\text {Loadi }}
$$

$\sum_{\mathrm{i}=1}^{\mathrm{N}} \mathrm{P}_{\text {Loadi }}=$ Total active load of the network

Now considering all constrains discussed above, and objective function considered the overall objective function will look like:

$$
\begin{aligned}
& \min \left[f_{n}\left(x_{s}, x_{c}\right)\right] \\
& \text { s.t. } h_{i}\left(x_{s}, x_{c}\right)=0, \quad i=1, \ldots, p \\
& \mathrm{~g}_{\mathrm{i}}\left(\mathrm{x}_{\mathrm{s}}, \mathrm{x}_{\mathrm{c}}\right) \leq 0, \quad \mathrm{i}=1, \ldots, \mathrm{q} \\
& \mathrm{x}_{\mathrm{S}}=\left[\begin{array}{c}
\mathrm{V}_{1} \\
\theta_{1} \\
\vdots \\
\mathrm{V}_{\mathrm{N}} \\
\theta_{\mathrm{N}}
\end{array}\right] \\
& \mathrm{X}_{\mathrm{C}}=\left[\begin{array}{c}
\mathrm{Loc}_{\mathrm{DG}_{1}} \\
\mathrm{P}_{\mathrm{DG}_{1}} \\
\vdots \\
\mathrm{Loc}_{\mathrm{DG}_{\mathrm{i}}} \\
\mathrm{P}_{\mathrm{DG}_{\mathrm{i}}} \\
\vdots \\
\mathrm{Loc}_{\mathrm{DG}_{\mathrm{N}}} \\
\mathrm{P}_{\mathrm{DG}} \\
\vdots
\end{array}\right]
\end{aligned}
$$

Where,

$\mathrm{f}_{\mathrm{n}}$ - Objective function $\quad \mathrm{h}_{\mathrm{i}}-$ Equality constrains

$g_{i}$ - Inequality constrains $x_{s}$ - Matrix of state variables

$x_{c}$ - Controllable variables

\section{III.DRAGON FLY ALGORITHM}

It can be observed that DG allocation and sizing problem is highly complex problem. With increase in the size of network, the problem becomes more complex and search space more limited, to solve this kind of problem Artificial Intelligent technique for optimization can be used.S. Mirjalili, [14] proposed a n ovel swarm intelligence optimization technique called dragonfly algorithm (DA). It is inspired by the static and dynamic swarming behaviours of dragonflies in nature. There are nearly 3000 different species of this fancy insect (Odonata) around the world. Their behaviour is governed by three principles: 


\section{IJIREEICE

1. Separation, which refers to the static collision avoidance of the individuals from other individuals in the neighbourhood.

2. Alignment, which indicates velocity matching of individuals to that of other individuals in neighbourhood.

3. Cohesion, which refers to the tendency of individuals towards the centre of the mass of the neighbourhood.

Two other factors are added based on the survival instincts of these insects, they tend to move towards the food source and move away from enemy, these behaviour are shown in Figure 1.

Apart from this there is another typical characteristic of these flies:

1. Hunting - static (feeding) swarm: Local movements and abrupt changes in the flying path.

2. Migration - dynamic (migratory) swarm: Massive number of dragonflies make the swarm for migrating in one direction.

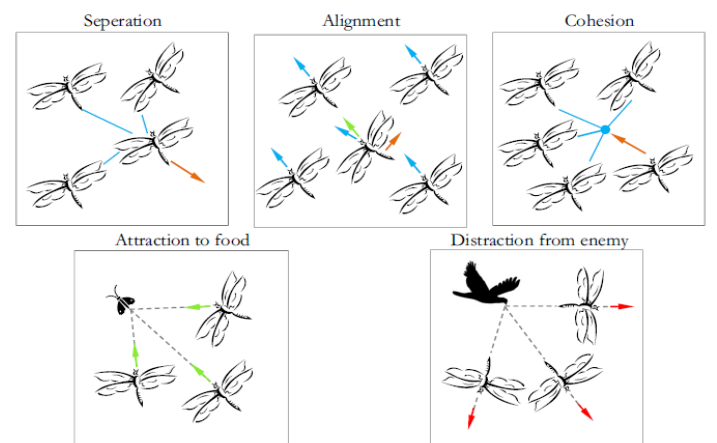

Fig. 1 Primitive corrective patterns between individuals. [14]

A. Mathematical model and algorithm

The above behaviour of Dragon flies is mathematically modelled and then optimization algorithm is developed.

1. Separation:

$$
S_{i}=-\sum_{j=1}^{N} X-X_{j}(12)
$$

Where $\mathrm{X}$ is the position of the current individual, $\mathrm{X}_{\mathrm{j}}$ shows the position $\mathrm{j}^{\text {th }}$ neighbouring individual, and $\mathrm{N}$ is the number of neighbouring individuals.

2. Alignment:

$$
A_{i}=\frac{\sum_{j=1}^{N} V_{j}}{N}(13)
$$

Where $X_{j}$ shows the velocity of $\mathrm{j}^{\text {th }}$ neighbouring individual.

3. Cohesion:

$$
C_{i}=\frac{\sum_{j=1}^{N} X_{j}}{N}-X(14)
$$

Where $\mathrm{X}$ is the position of the current individual, $\mathrm{N}$ is the number of neighbourhoods, and $\mathrm{X}_{\mathrm{j}}$ shows the position $\mathrm{j}^{\text {th }}$ neighbouring individual

4. Attraction to food:

Attraction towards a food source is calculated as follows:

$$
F_{i}=X^{+}-X(15)
$$

Where $\mathrm{X}$ is the position of the current individual, and $\mathrm{X}^{+}$shows the position of the food source.

4. Distraction from enemy:

Distraction outwards an enemy is calculated as follows:

$$
E_{i}=X^{-}+X(16)
$$




\section{International Journal of Innovative Research in Electrical, Electronics, Instrumentation and Control Engineering \\ ISO 3297:2007 Certified \\ Vol. 5, Issue 4, April 2017}
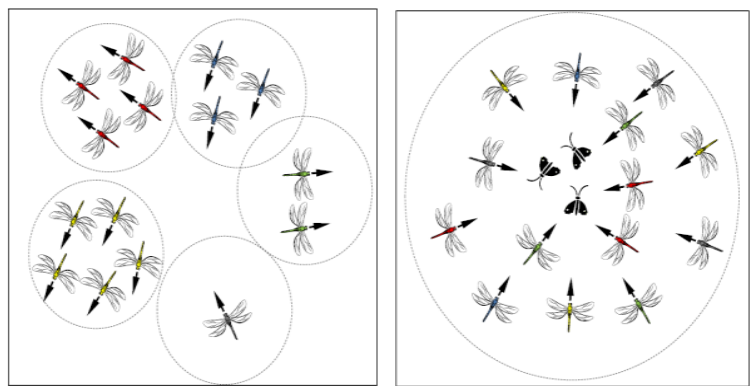

Fig. 2 Dynamic and Static swarm behaviours. [14]

Where $\mathrm{X}$ is the position of the current individual, and $\mathrm{X}^{-}$shows the position of the enemy.

These five patterns determine the behaviour of dragonflies. The position of artificial dragonflies in a search space is updated by equation given bellow.

$$
\Delta \mathrm{X}_{\mathrm{t}+1}=\left(\mathrm{sS}_{\mathrm{i}}+\mathrm{aA} \mathrm{A}_{\mathrm{i}}+\mathrm{cC}_{\mathrm{i}}+\mathrm{fF}_{\mathrm{i}}+\mathrm{eE}_{\mathrm{i}}\right)+\mathrm{w} \Delta \mathrm{X}_{\mathrm{t}}(17)
$$

Where each coefficient represents the weights for the respective characteristics vector. Now position for next step can be determined by:

$$
\mathrm{X}_{\mathrm{t}+1}=\mathrm{X}_{\mathrm{t}}+\Delta \mathrm{X}_{\mathrm{t}+1}(18)
$$

5. Static and Dynamic behaviour of Dragon flies:

In a static swarm, however, alignments is kept very low while cohesion is high (attacking mode). Therefore we can explore or exploit the search space by choosing proper alignment and cohesion weights. For this the radii of neighbourhoods are increased proportional to the number of iterations. Thus with increase in number of iterations the flies will transit from exploration to exploitation by changing the values of individual weights iteratively. Le'vy flight is employed to incorporate the random behaviour of flies when there are no neighbouring solutions. It is given by following equations:

$$
X_{t+1}=X_{t}+\operatorname{Levy}(d) \times X_{t}(19)
$$

Where $t$ is the current iteration and $\mathrm{d}$ is the dimension of the position vectors.

The Le'vy flight is calculated as follows:

$$
\operatorname{Levy}(\mathrm{x})=0.01 \times \frac{\mathrm{r}_{1} \times \sigma}{\left|\mathrm{r}_{2}\right|^{\frac{1}{\beta}}}(20)
$$

Where $r_{1}, r_{2}$ are random numbers between [0,1], b is a constant (equal to 1.5 in this work), and $r$ is calculated as follows:

$$
\sigma=\left(\frac{\Gamma(1+\beta) \times \sin \left(\frac{\pi \beta}{2}\right)}{\Gamma\left(\frac{1+\beta}{2}\right) \times \beta \times 2^{\left(\frac{\beta-1}{2}\right)}}\right)^{1 / \beta}
$$

Where, $\Gamma(\mathrm{x})=(\mathrm{x}-1)$ !

\section{IV.CASE STUDY ON IEEE 33 BUS SYSTEM}

IEEE 33 bus test case [2] was considered to demonstrate the performance of DA algorithm. MATLAB (2012) code was developed to obtain the optimal value of control parameters and MATPOWER 5.0 software package was used to obtain power flow solution. The objective functions considered here are the total power losses, voltage deviation and voltage stability margin. The summary of the test case is given in table I, while the detail data such as line data and load data are given in [2] The program is implemented on a personal computer with Intel i3 quad core $2.4 \mathrm{GHz}$ processor and $4 \mathrm{~GB}$ total memory.

TABLE I SUMMARY OF TEST CASE

\begin{tabular}{|c|c|}
\hline Specification & IEEE 33 bus \\
\hline Bus voltage & $11 \mathrm{kV}$ \\
\hline Lines & 32 \\
\hline Generators & 1 \\
\hline Transformer & 0 \\
\hline
\end{tabular}


ISO 3297:2007 Certified

Vol. 5, Issue 4, April 2017

\begin{tabular}{|c|c|}
\hline Loads & 32 \\
\hline Active Load & $3.715 \mathrm{MW}$ \\
\hline
\end{tabular}

CASE 1: DA programme to solvepower loss minimization problem was set to run for 100 iterations and with 20 search agents and total 20 trials were taken. We can observe that the total losses for best solution obtained are reduced to 0.0873 MW from 0.2027 MW (without DG placement). The load flow results for best solution obtained are shown in table II.

TABLE II RESULTS OBTAINED FOR THE BEST OF 20 SOLUTIONS (CASE 1)

\begin{tabular}{|c|c|c|}
\hline \multicolumn{2}{|c|}{ DG planning Scheme } & Objective function \\
\hline Position & Capacity (MW) & Power loss (MW) \\
\hline 18 & 0.3923 & \multirow{2}{*}{0.0873} \\
\hline 33 & 0.5365 & \\
\hline \multicolumn{2}{|c|}{ Time taken } & $14.8 \mathrm{sec}$ \\
\hline \multicolumn{3}{|c|}{ Without optimization } \\
\hline - & - & 0.2027 \\
\hline
\end{tabular}

As we increase the number of iteration and number of search agents we get more accurate results with better convergence curve, but with this increase in iteration count and population size, the number of NR power flow problem to be solved increases by great extent, thus we need to maintain balance between these two. Further it is observed that with increase in number of decision variables the complexity of problem increases significantly.

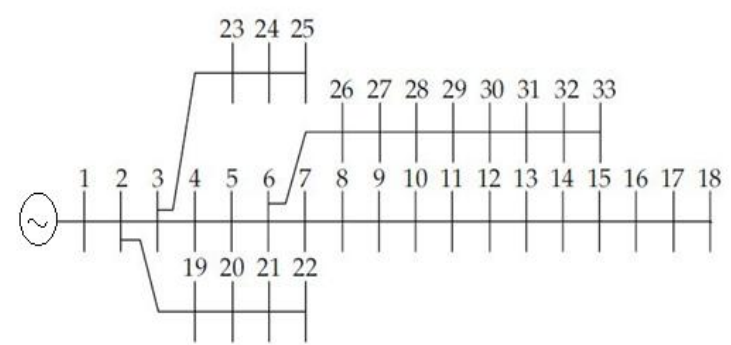

Fig. 3 IEEE 33 bus system single line diagram. [2]

CASE 2: Here multi-objective optimization function is considered where both active power losses as well as voltage deviation at each bus has been minimized. The method used is weighted sum of each objective to minimize the objective function. The program was set to run for 100 iterations and with 20 search agents and total 20 trials were taken. The load flow results for best solution obtained are shown in table III.

TABLE III RESULTS OBTAINED FOR THE BEST OF 20 SOLUTIONS (CASE II)

\begin{tabular}{|c|c|c|c|}
\hline \multicolumn{2}{|c|}{ DG planning Scheme } & \multicolumn{2}{|c|}{ Objective function } \\
\hline Position & Capacity (MW) & Power loss (MW) & Voltage Deviation \\
\hline 18 & 0.4991 & \multirow{2}{*}{0.0893} & \multirow{2}{*}{0.7181} \\
\hline 33 & 0.4247 & & \\
\hline \multicolumn{2}{|c|}{ Time taken } & \multicolumn{2}{|c|}{$15 \mathrm{sec}$} \\
\hline \multicolumn{4}{|c|}{ Without optimization } \\
\hline- & - & 0.2027 & 11.7097 \\
\hline
\end{tabular}

CASE 3: Here another objective function - maximization of voltage stability margin is incorporated for our test case. The method used is again weighted sum of each objective method to minimize the objective function and penalty is added if there are any violations in constraints. $\mathrm{L}$ index is calculated for each possible solution and the results summary for best solution is shown in table IV.

TABLE IV RESULTS OBTAINED FOR THE BEST OF 20 SOLUTIONS (CASE III)

\begin{tabular}{|c|c|c|c|c|}
\hline \multicolumn{2}{|c|}{ DG planning Scheme } & \multicolumn{3}{c|}{ Objective function } \\
\hline Position & Capacity (MW) & Power loss (MW) & Voltage Deviation & Voltage Stability index \\
\hline
\end{tabular}


ISO 3297:2007 Certified

Vol. 5, Issue 4, April 2017

\begin{tabular}{|c|c|c|c|c|}
\hline 15 & 0.3630 & \multirow{2}{*}{0.0997} & \multirow{2}{*}{0.4444} & \multirow{2}{*}{0.0287} \\
\hline 33 & 0.5658 & & & \\
\hline \multicolumn{2}{|c|}{ Time taken } & \multicolumn{3}{|c|}{$15.8 \mathrm{sec}$} \\
\hline \multicolumn{5}{|c|}{ Without optimization } \\
\hline- & - & 0.2027 & 11.7097 & 0.0746 \\
\hline
\end{tabular}

When we compare these results with those found in literature we can see that performance of DA optimization technique is satisfactory.

TABLE V COMPARISON WITH RESULTS FOUND IN LITERATURE

\begin{tabular}{|c|c|c|c|c|c|c|}
\hline & \multirow{2}{*}{ Harmony search[8] } & \multirow{2}{*}{ INSGA[7] } & \multirow{2}{*}{ MSFL[15] } & \multicolumn{3}{|c|}{ Dragonfly } \\
\cline { 5 - 7 } & & & CASE 1 & CASE II & CASE III \\
\hline Power loss & 0.1016 & 0.1042 & 0.092 & 0.087 & 0.089 & 0.099 \\
\hline Voltage deviation & 3.7405 & 3.7282 & 0.996 & 0.821 & 0.718 & 0.444 \\
\hline Voltage Stability index & 0.0528 & 0.0554 & - & - & - & 0.028 \\
\hline Time & - & $33.49 \mathrm{sec}$ & & $14.8 \mathrm{~s}$ & $15 \mathrm{~s}$ & $15.8 \mathrm{~s}$ \\
\hline
\end{tabular}

\section{CONCLUSION}

The Optimization problem formulated is very complex and highly non-linear, any conventional method will take huge amount of time and because of several approximations used in these methods we might not get accurate results. Artificial intelligent techniques has an advantage over this, thus dragonfly algorithm is used.Further to check performance of DA to solve power system optimization problems, this method was implemented on IEEE 33 bus test case. The objective functions considered were active power loss minimization, voltage stability margin improvement and minimization of voltage deviation.Penalty functions were used to convert constrained optimization problem to unconstrained one.Results had been compared to that found in literature and it is observed that DA gives competitive results as compared to other methods, while it also outperforms other algorithms in few cases.

\section{REFERENCES}

[1] Thomas Ackermann, ValeyKnyazkin "Interaction between Distributed Generation and Distribution Network: Operation Aspects", Transmission and Distribution Conference and Exhibition 2002: Asia Pacific. IEEE/PES, vol. 2, pp. 1357- 1362, Oct 2002, IEEE.

[2] A. Kazemi, M. Sadeghi, "Distributed Generation Allocation for Loss Reduction and Voltage Improvement", Power and Energy Conference, APPEEC 2009. Asia-Pacific, pp. 1-6, March 2009, IEEE.

[3] N.S. Rau, Y.-H. Wan, "Optimum location of resources in distributed planning," IEEE Transactions on Power Systems, vol. 9, no. 4, 1994, p. 2014-2020.

[4] M.F. AlHajri, M.E. El-Hawary, "Optimal distributed generation siting via fast sequential quadratic programming", Proceedings of Large Engineering Systems Conference on Power Engineering, 2007, pp. 63-66.

[5] M.R. Vallem, J. Mitra, "Siting and sizing of distributed generation for optimal microgrid architecture," Proceedings of 37th Annual North American Power Symposium, 2005, pp.611- 616.

[6] S. Kamalinia, S. Afsharnia, M.E. Khodayar, A. Rahimikian, M.A. Sharbafi, "A combination of MADM and genetic algorithm for optimal DG allocation in power systems," Proceedings of $42^{\text {nd }}$ University Power Engineering Conference, 2007, pp. 1031-1035.

[7] Sheng, Wanxing, et al. "Optimal placement and sizing of distributed generation via an improved nondominated sorting genetic algorithm II." IEEE Transactions on Power Delivery 30.2 (2015): 569-578.

[8] Liu, Yuan, et al. "Optimal placement and sizing of distributed generation in distribution power system based on multi-objective harmony search algorithm." 2013 6th IEEE Conference on Robotics, Automation and Mechatronics (RAM).IEEE, 2013.

[9] Roy, Santanu, Sneha Sultana, and Provas Kumar Roy. "Oppositional cuckoo optimization algorithm to solve DG allocation problem of radial distribution system."Recent Developments in Control, Automation and Power Engineering (RDCAPE), 2015 International Conference on.IEEE, 2015.

[10] Candelo, J. E., and H. E. Hernández. "Location and Size of Distributed Generation to Reduce Power Losses using a Bat-inspired Algorithm."VII SimposioInternacionalsobreCalidad de la EnergíaEléctrica. 2013.

[11] W. EI-Khattam, K. Bhattacharya, YHegazy, M. M. A. Salama, "Optimal nvestment Planning for Distributed Generation in a Competitive Electricity Market," IEEE Transactions on Power Systems, volume 19, issue 3

[12] K. Nara, Y. Hayashi, K. Ikeda, T. Ahizawa, "Application of Tabu Search to Optimal Placement of Distributed Generators", Proceedings of the IEEE Power Engineering Society Winter Meeting, 2001

[13] Falaghi, H. ,Haghi, M. R. , "ACO based algorithm for distributed generation sources allocation and sizing in distribution systems," IEEE Power Tech conference, 2007

[14] Mirjalili, Seyedali. "Dragonfly algorithm: a new meta-heuristic optimization technique for solving single-objective, discrete, and multi-objective problems." Neural Computing and Applications 27.4 (2016): 1053-1073.

[15] Mistry, Khyati. "MSFL based determination of optimal size and location of distributed generation in radial distribution system." Electrical, Electronics, and Optimization Techniques (ICEEOT), International Conference on.IEEE, 2016. 